Article

\title{
Natural Product-Based Pesticide Discovery: Design, Synthesis and Bioactivity Studies of $N$-Amino-Maleimide Derivatives
}

\author{
Xiangmin Song ${ }^{1}$, Chunjuan Liu ${ }^{2}$, Peiqi Chen ${ }^{2}$, Hao Zhang ${ }^{2}$ and Ranfeng Sun ${ }^{1, *}$ \\ 1 Institute of Tropical Agriculture and Forestry, Hainan University, Haikou 570228, China; \\ 18898958572@163.com \\ 2 School of Chemical \& Chemical Engineering, Inner Mongolia University, Hohhot 010021, China; \\ 17330926558@163.com (C.L.); chenpq17@1zu.edu.cn (P.C.); haozhang@imu.edu.cn (H.Z.) \\ * Correspondence: srf18@hainu.edu.cn; Tel.: +86-898-6619-2906
}

Received: 31 May 2018; Accepted: 21 June 2018; Published: 24 June 2018

\begin{abstract}
Natural products are an important source of pesticide discovery. A series of $\mathrm{N}$-amino-maleimide derivatives containing hydrazone group were designed and synthesized based on the structure of linderone and methyllinderone which were isolated from Lindera erythrocarpa Makino. According to the bioassay results, compounds 2 and 3 showed $60 \%$ inhibition against mosquito (Culex pipiens pallens) at $0.25 \mu \mathrm{g} \cdot \mathrm{mL}^{-1}$. Furthermore, the results of antifungal tests indicated that most compounds exhibited much better antifungal activities against fourteen phytopathogenic fungi than linderone and methyllinderone and some compounds exhibited better antifungal activities than commercial fungicides (carbendazim and chlorothalonil) at $50 \mu \mathrm{g} \cdot \mathrm{mL}^{-1}$. In particular, compound 12 exhibited broad-spectrum fungicidal activity ( $>50 \%$ inhibitory activities against 11 phytopathogenic fungi) and compounds 12 and $\mathbf{1 4}$ displayed $60.6 \%$ and $47.9 \%$ inhibitory activity against Rhizoctonia cerealis at $12.5 \mu \mathrm{g} \cdot \mathrm{mL}^{-1}$ respectively. Furthermore, compound $\mathbf{1 7}$ was synthesized, which lacks $\mathrm{N}$-substituent at maleimide and its poor antifungal activity against Sclerotinia sclerotiorum and Rhizoctonia cerealis at $50 \mu \mathrm{g} \cdot \mathrm{mL}^{-1}$ showed that the backbone structure of $\mathrm{N}$-amino-maleimide derivatives containing hydrazone group was important to the antifungal activity.
\end{abstract}

Keywords: maleimide; linderone; methyllinderone; antifungal activity

\section{Introduction}

Chitin is a unique component of the fungal cell wall and shells of crustaceans, but it is absent in vertebrates, mammals, and humans [1]. Chitin synthase is thus an attractive molecular target for developing fungicides and insecticides. Natural products are an important source for drug and pesticide discovery. Lindera species (Lauraceae) have rich chemical compositions and pharmacological activities [2]. Methyllinderone (A) and linderone (B), which were classic inhibitors of chitin synthetase (Figure 1), were isolated from Lindera erythocarpa Makino (Lauraceae) and exhibited inhibitory activity against chitin synthase 2 (CaCHS2p) with $\mathrm{IC}_{50}$ value of $23.3,21.4 \mu \mathrm{g} \cdot \mathrm{mL}^{-1}$ respectively, which was better than polyoxin $\mathrm{D}\left(70.0 \mu \mathrm{g} \cdot \mathrm{mL}^{-1}\right)$ and nikkomycin $\mathrm{Z}\left(176.0 \mu \mathrm{g} \cdot \mathrm{mL}^{-1}\right)$ [3]. In 2015 , Seok-Hee Lee et al. reported that methyllinderone (A) and methyllucidone (C) (Figure 1) exhibited juvenile hormone antagonistic activity against Aedes aegypti [4]. Sheng-Yang Wang et al. reported that linderone (D), methyllinderone (A), lucidone (E) and methyllucidone (C) (Figure 1) displayed good anti-inflammatory activity [5]. 


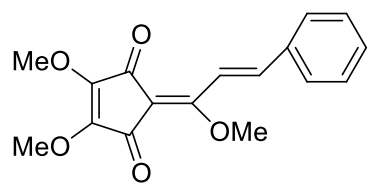

methyllinderone ( A )<smiles>COC1=CC(=O)C(=C(C=Cc2ccccc2)OC)C1=O</smiles>

methyllucidone ( C )<smiles>COC1=CC(=O)C(=C(O)C=Cc2ccccc2)C1=O</smiles><smiles>COC1=C(OC)C(=O)C(C(=O)/C=C/c2ccccc2)C1=O</smiles>

linderone ( B )<smiles>COC1=C(OC)C(=O)C(=C(O)C=Cc2ccccc2)C1=O</smiles>

linderone (D)

lucidone ( E )

Figure 1. Chemical structures of methyllinderone (A), linderone (B), methyllucidone (C), linderone (D), lucidone (E).

Interestingly, it was found that compounds containing a maleimide fragment also have chitin synthase and $\beta$-1,3-glucan synthase inhibitory activity according to the literature. For example, compounds $\mathbf{F}$ and $\mathbf{G}$ (Figure 2) inhibited $\beta$-1,3-glucan synthase with $\mathrm{IC}_{50}$ value of $8.5,17.3 \mu \mathrm{g} \cdot \mathrm{mL}^{-1}$, respectively [6]. In 2007, Atul R. Gholap et al. reported compound $\mathbf{H}$ (Figure 2) containing maleimide group performed over $91 \%$ inhibitory against chitin synthase, which exhibited comparable activity with nikkomycin Z (90\%) [7].<smiles>CCN1C(=O)C=CC1=O</smiles>

$\mathrm{F}$<smiles>CC(C)(C)N1C(=O)C=CC1=O</smiles>

G<smiles>[R]Nc1cc2c(c(-c3ccccc3)c1C#N)C(=O)CCC2</smiles>

a<smiles></smiles>
$\mathrm{R}_{1}=\mathrm{H}$

$\mathrm{H}$<smiles>[R]=COC=[R]</smiles>

Figure 2. Structures of compounds with maleic amide fragment. 
Therefore, we focused on introducing maleimide fragments into natural products to obtain more efficient fungicides and insecticides. A series of $\mathrm{N}$-amino-maleimide derivatives containing hydrazone group were designed based on the structure of methyllinderone, linderone and acylhydrazone derivatives with excellent antifungal activity (Figure 3) [8]. Then target compounds 1-16 were synthesized and their biological activities were evaluated.
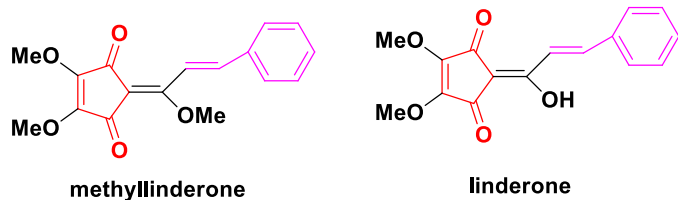

chitin synthase inhibitors

Planta Med. 2007, 73, 679-682
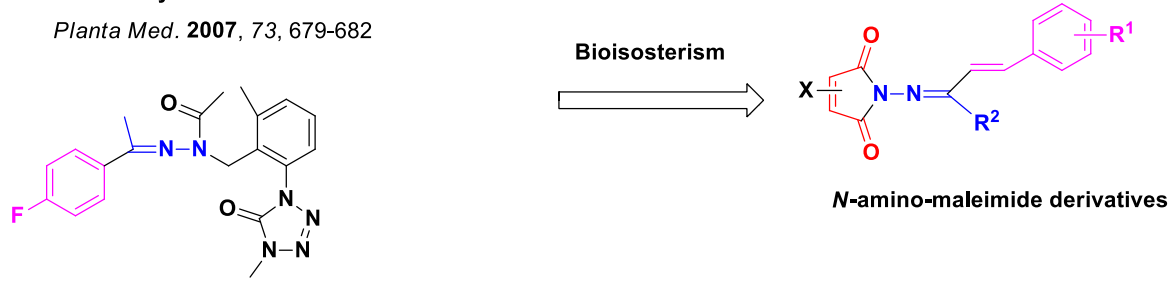

acylhydrazone derivatives with antifungal activities

JP 2016204377

Figure 3. Design strategy for $\mathrm{N}$-amino-maleimide derivatives.

\section{Results}

\subsection{Synthesis}

Different aryl-substituted unsaturated ketones, which were synthesized according to the literature [9], and $\mathrm{N}$-amino-maleimide, which were produced in situ without purification [10], are refluxed in dry EtOH with a catalytic amount of $p$-toluenesulfonic acid to produce a variety of $\mathrm{N}$-amino-maleimide derivatives containing hydrazone group (compounds $\mathbf{1 - 1 6}$, Scheme 1 ) and NMR spectral information was listed (Figures S1-S34). Although the reaction produced good yields, the maleic anhydride and hydrazine hydrate cyclized to form a six-membered ring isomer (Figure 4) which is in accordance with previous report [10].

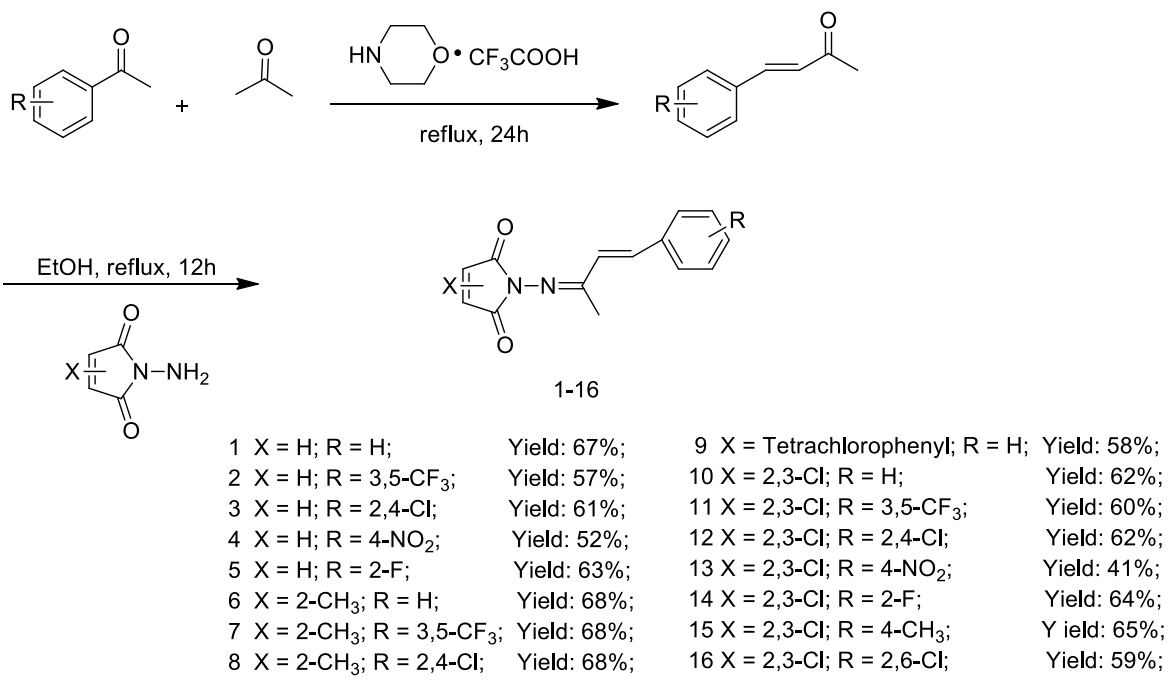

Scheme 1. General synthetic route for compounds 1-16. 


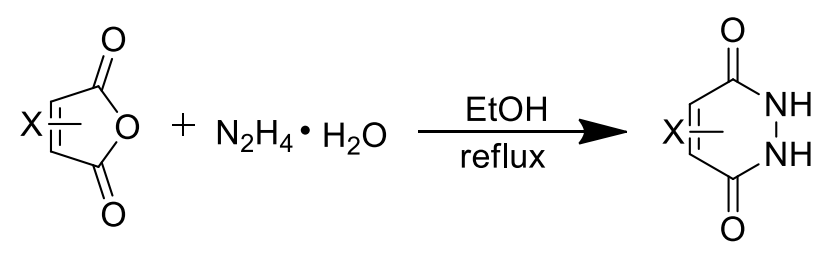

Figure 4. Synthesis of six-membered ring isomers.

At the same time, linderone and methyllinderone were synthesized according to the literature (Scheme 2) [11].
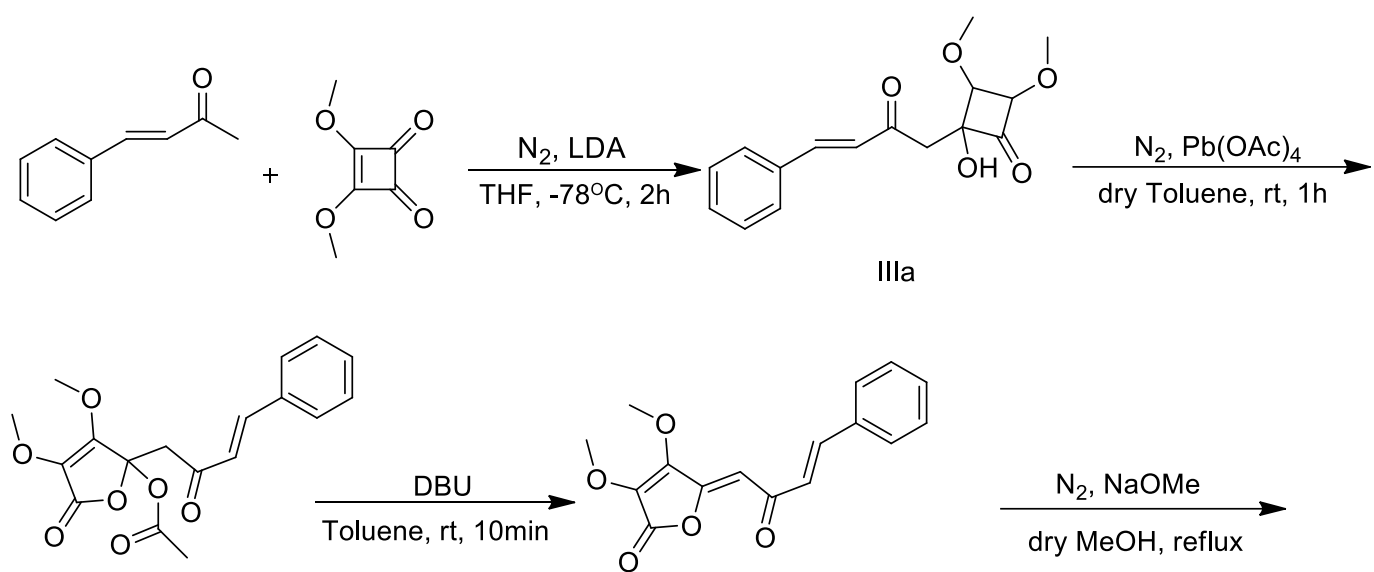

IIIb

IIIc<smiles>COC1=C(OC)C(=O)C(=C(O)C=Cc2ccccc2)C1=O</smiles>

$\mathrm{Me}_{2} \mathrm{SO}_{4}, \mathrm{~K}_{2} \mathrm{CO}_{3}$, acetone<smiles>COC(/C=C/c1ccccc1)=C1C(=O)C(OC)=C(OC)C1=O</smiles>

Linderone

Yield: $84 \%$

Methyllinderone Yield: $93 \%$

Scheme 2. Synthetic route for linderone and methyllinderone.

Finally, 3,4-dichloro-1H-pyrrole-2,5-dione (17) was synthesized to further illustrate the active functional group of compounds 1-16 (Scheme 3) [10].<smiles>C[Si](C)(C)N[Si](C)(C)C</smiles><smiles></smiles><smiles>O=C1NC(=O)C(Cl)=C1Cl</smiles>

17 Yield: $82 \%$

Scheme 3. Synthetic route for compound 17. 


\subsection{Bioassays}

\subsubsection{Stomach Toxicity against Oriental Armyworm (Mythimna separata)}

It can be seen from Table 1 that some compounds have considerable insecticidal activities against oriental armyworm and seemed to display some electronic effects. For example, comparing the insecticidal activities of compounds $1,6,10$, we can easily find that when the phenyl ring $(R=H)$ is unsubstituted, the electron-rich substituents of maleimide unit will significantly enhance the insecticidal activity of the compound, the insecticidal activity values of compounds $\mathbf{1}(X=H)$, $6\left(X=2-\mathrm{CH}_{3}\right), 10(X=2,3-\mathrm{Cl})$ are $25 \%, 65 \%$ and $20 \%$ respectively. When comparing two sets of the compounds substituted in maleinimide fragment $\mathbf{1 - 5}(\mathrm{X}=\mathrm{H})$ or $\mathbf{6}-\mathbf{8}\left(\mathrm{X}=\mathbf{2}-\mathrm{CH}_{3}\right)$, it was found that they displayed different structure-activity relationships $\left(S A R_{S}\right)$. For example, when the maleimide units are unsubstituted $(X=H)$, compound $2\left(R=3,5-C_{3}\right)$ and $3(R=2,4-C l)$ displayed superior insecticidal activities $\left(60 \%, 50 \%\right.$ respectively) than compound $4\left(\mathrm{R}=4-\mathrm{NO}_{2}\right)$ and $5(\mathrm{R}=2-\mathrm{F})(10 \%$, $25 \%$ respectively), whereas compound $6(\mathrm{R}=\mathrm{H})$ exhibited the best insecticidal activities when the substituent of maleimide units is methyl group $\left(\mathrm{X}=2-\mathrm{CH}_{3}\right)$. In general, the insecticidal activity of the meta-substitute on the phenyl ring is superior to that of the ortho and para-substitute.

Table 1. Larvacidal activities against oriental armyworm and mosquito of compounds 1-8, 10.

\begin{tabular}{cccccccc}
\hline & \multicolumn{7}{c}{ Larvicidal Activity $(\%)$ at Concn $\left(\boldsymbol{\mu g} \cdot \mathbf{m L}^{-\mathbf{1}}\right)$} \\
\cline { 2 - 8 } Compd. & Oriental Armyworm & \multicolumn{7}{c}{ Mosquito } \\
\cline { 2 - 8 } & $\mathbf{6 0 0}$ & $\mathbf{1 0}$ & $\mathbf{5}$ & $\mathbf{2}$ & $\mathbf{1}$ & $\mathbf{0 . 5}$ & $\mathbf{0 . 2 5}$ \\
\hline $\mathbf{1}$ & 25 & 50 & - a & - & - & - & - \\
$\mathbf{2}$ & 60 & 100 & 100 & 100 & 100 & 100 & 60 \\
$\mathbf{3}$ & 50 & 100 & 100 & 100 & 100 & 100 & 60 \\
$\mathbf{4}$ & 10 & 40 & - & - & - & - & - \\
$\mathbf{5}$ & 25 & 30 & - & - & - & - & - \\
$\mathbf{6}$ & 65 & 50 & - & - & - & - & - \\
$\mathbf{7}$ & 40 & 100 & 20 & - & - & - & - \\
$\mathbf{8}$ & 5 & 100 & 100 & 100 & 100 & 100 & 20 \\
$\mathbf{1 0}$ & 20 & 100 & 100 & 40 & - & - & - \\
\hline
\end{tabular}

\subsubsection{Toxicity against Mosquito (Culex pipiens pallens)}

Table 1 showed the larvacidal activities of the compounds 1-8, 10 against mosquito. The bioassay results indicated that some compounds (compounds 2, 3 and 8) exhibited excellent larvicidal activities against mosquito, especially compounds $2\left(X=H, R=3,5-C_{3}\right)$ and $3(X=H, R=2,4-C l)$ which showed $60 \%$ mortality even at $0.25 \mu \mathrm{g} \cdot \mathrm{mL}^{-1}$. This seems to indicate that the substitution of the meta position on the phenyl ring or the substitution of the ortho and para positions will have a greater effect on the activity of the compounds.

\subsubsection{In Vitro Antifungal Activity}

The fungicidal results of compounds 1-17 are listed in Tables 2 and 3. Most compounds exhibited much better antifungal activities against fourteen phytogenic fungi than linderone and methyllinderone and some compounds exhibited better antifungal activities than commercial fungicides (carbendazim and chlorothalonil). All the compounds except compound 1, 4-6 and 9 showed more than $50 \%$ inhibition rate against Rhizoctonia cerealis at $50 \mu \mathrm{g} \cdot \mathrm{mL}^{-1}$ and six compounds (10-13, 14-16) showed better antifungal activities than linderone (58.2\%) and methyllinderone (74.5\%), in particular compounds 12, 14, and 15 with $98.2 \%, 100.0 \%, 90.9 \%$ of inhibition rate respectively. When the concentration was adjusted to $25 \mu \mathrm{g} \cdot \mathrm{mL}^{-1}$ and $12.5 \mu \mathrm{g} \cdot \mathrm{mL}^{-1}$, the inhibition rate of 
compound 12 against Rhizoctonia cerealis was $95.8 \%, 60.6 \%$ and the inhibition rate of compound 14 against Rhizoctonia cerealis was 77.5\%, 47.9\% respectively (Table 3). Compound 2, 4, 6, 7, 10, 14 and 16 showed more than $50 \%$ inhibition rate against Botryospuaeria berengeriana at $50 \mu \mathrm{g} \cdot \mathrm{mL}^{-1}$, and five of them (compound 2, 4, 6, 10 and 14) were superior to linderone $(57.1 \%)$ and methyllinderone (8.2\%), for example, compound 4 exhibited 89.3\% inhibitory activity. Compound 10-12 and 14-16 showed more than $60 \%$ inhibition rate against Sclerotinia sclerotiorum at $50 \mu \mathrm{g} \cdot \mathrm{mL}^{-1}$, which were even superior to chlorothalonil (<50\%). Compound 8, 11, 12, 15 and 16 showed more than 50\% inhibition rate against Cercospora arachidicola at $50 \mu \mathrm{g} \cdot \mathrm{mL}^{-1}$, which were even superior to carbendazim $(<50 \%)$. Compound 4, 12, 15 and 16 displayed more than 50\% inhibition rate against Helminthosporium maydis at $50 \mathrm{\mu g} \cdot \mathrm{mL}^{-1}$, which were superior to linderone (27.8\%) and methyllinderone (38.9\%). Compound 10-12 and 15-16 showed more than 50\% inhibition rate against Botrytis cinerea at $50 \mu \mathrm{g} \cdot \mathrm{mL}^{-1}$, which were superior to linderone (27.8\%) and methyllinderone (38.9\%). Compound 2, 7, 10, 12 and 14-16 showed more than $50 \%$ inhibition rate against Rhizoctonia solani at $50 \mu \mathrm{g} \cdot \mathrm{mL}^{-1}$, which were superior to linderone (28.4\%) and methyllinderone (24.7\%). Compound 12 and 14-16 showed more than 50\% inhibition rate against Phytophthora capsica at $50 \mu \mathrm{g} \cdot \mathrm{mL}^{-1}$, which were superior to linderone $(27.8 \%)$ and methyllinderone (27.8\%). In particular, compound 14 exhibited broad-spectrum fungicidal activities against test phytogenic fungi and even displayed much better antifungal activities against five fungi (Botrytis cinereal, Fusarium oxysporum f.sp. cucumerinum, Cercospora arachidicola, Fusarium moniliforme and Phytophthora capsica) than carbendazim $(<50 \%)$. At the same time, we found that the substituents of maleimide have an important effect on the antifungal activity, for example, compound $12(X=2,3-\mathrm{Cl}$, $\mathrm{R}=2,4-\mathrm{Cl})$ exhibits more power and broad-spectrum antifungal activity than compounds $3(X=H$, $\mathrm{R}=2,4-\mathrm{Cl}), 8\left(\mathrm{X}=2-\mathrm{CH}_{3}, \mathrm{R}=2,4-\mathrm{Cl}\right)$. Finally, in order to prove whether the maleimide has antifungal activity as reported in the literature [12], we synthesized compound $\mathbf{1 7}$ and then tested their inhibitory activities against Sclerotinia sclerotiorum and Rhizoctonia cerealis, which was $21.4 \%, 23.6 \%$ respectively at $50 \mu \mathrm{g} \cdot \mathrm{mL}^{-1}$ (Table 3). This result indicates that the antifungal activities of compounds 1-16 are dependent by the entire pesticide molecule rather than the maleimide fragment.

Table 2. Fungicidal activity of title compounds 1-16 and Linderone, Methyllinderone at $50 \mu \mathrm{g} \cdot \mathrm{mL}^{-1}$.

\begin{tabular}{|c|c|c|c|c|c|c|c|c|c|c|c|c|c|c|}
\hline \multirow{2}{*}{ Compd. } & \multicolumn{14}{|c|}{ Inhibition Rate (\%) } \\
\hline & A.S ${ }^{a}$ & G.Z & P.I & $S . S$ & B.C & R.S & F.O & C.A & B. B & R.C & H.M & $C . L$ & F.M & P.C \\
\hline 1 & 25.0 & 17.2 & 17.6 & 8.9 & 15.4 & 6.2 & 11.6 & 36.7 & 26.8 & 34.9 & 32.7 & 10.0 & 18.2 & $-b$ \\
\hline 2 & 33.3 & 20.7 & 23.5 & 37.5 & 34.6 & 54.3 & 23.3 & 26.7 & 66.1 & 50.0 & 32.7 & 22.5 & 30.3 & - \\
\hline 3 & 8.3 & 24.1 & 17.6 & 17.9 & 26.9 & 37.0 & 32.6 & 46.7 & 35.7 & 57.0 & 34.7 & 32.5 & 57.6 & - \\
\hline 4 & 25 & 37.9 & 29.4 & 26.8 & 34.6 & 18.5 & 27.9 & 46.7 & 89.3 & 29.1 & 61.2 & 27.5 & 39.4 & - \\
\hline 5 & 8.3 & 10.3 & 17.6 & 8.9 & 7.7 & 12.3 & 14.0 & 36.7 & 25.0 & 31.4 & 30.6 & 5.0 & 15.2 & - \\
\hline 6 & 25 & 13.8 & 11.8 & 32.1 & 19.2 & 30.9 & 16.3 & 13.3 & 62.5 & 23.3 & 20.4 & 15.0 & 30.3 & - \\
\hline 7 & 16.7 & 10.3 & 23.5 & 21.4 & 19.2 & 53.1 & 20.9 & 36.7 & 51.8 & 58.1 & 26.5 & 22.5 & 30.3 & - \\
\hline 8 & 16.7 & 17.2 & 17.6 & 39.3 & 23.1 & 45.7 & 25.6 & 56.7 & 48.2 & 64.0 & 34.7 & 32.5 & 27.3 & - \\
\hline 9 & 33.3 & 44.8 & 23.5 & 5.4 & 3.8 & 14.8 & 16.3 & 30.0 & 7.1 & 40.7 & 26.5 & 17.5 & 21.2 & - \\
\hline 10 & 41.7 & 44.8 & 58.8 & 75.0 & 73.1 & 66.7 & 39.5 & 46.7 & 75.0 & 79.1 & 44.9 & 47.5 & 48.5 & - \\
\hline 11 & 27.8 & 39.5 & 18.2 & 63.4 & 52.4 & 43.2 & 45.7 & 50.0 & 14.3 & 89.1 & 47.2 & 40.7 & 46.2 & 33.3 \\
\hline 12 & 44.4 & 47.4 & 50.0 & 87.5 & 71.4 & 67.9 & 65.7 & 65.0 & 42.9 & 98.2 & 63.9 & 70.4 & 61.5 & 55.6 \\
\hline 13 & 44.4 & 36.8 & 27.3 & 26.8 & 47.6 & 42 & 51.4 & 35.0 & 34.7 & 61.8 & 27.8 & 18.5 & 38.5 & 27.8 \\
\hline 14 & 44.4 & 39.5 & 54.5 & 60.6 & 47.6 & 67.9 & 45.7 & 25.0 & 67.3 & 100.0 & 55.6 & 44.4 & 46.2 & 69.4 \\
\hline 15 & 44.4 & 44.7 & 63.6 & 66.2 & 52.4 & 63.0 & 45.7 & 50.0 & 36.7 & 90.9 & 52.8 & 51.9 & 46.2 & 69.4 \\
\hline 16 & 44.4 & 42.1 & 45.5 & 63.4 & 52.4 & 61.7 & 45.7 & 55.0 & 51.0 & 89.1 & 52.8 & 37.0 & 53.8 & 63.9 \\
\hline Linderone & 27.8 & 31.6 & 27.3 & 66.2 & 47.6 & 28.4 & 51.4 & 30.0 & 57.1 & 74.5 & 27.8 & 33.3 & 26.9 & 27.8 \\
\hline Methyllinderon & 38.9 & 31.6 & 27.3 & 42.3 & 42.9 & 24.7 & 34.3 & 20.0 & 8.2 & 58.2 & 38.9 & 33.3 & 38.5 & 27.8 \\
\hline
\end{tabular}


Table 2. Cont.

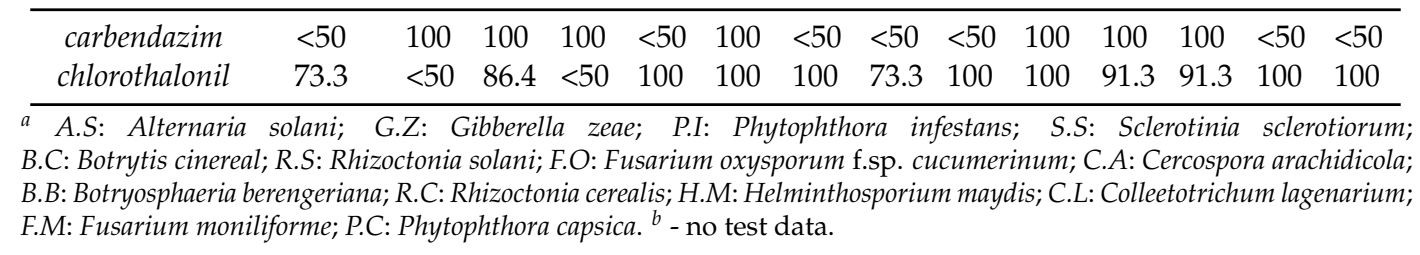

Table 3. Fungicidal activity of compounds 12, 14, 17 against $S$. sclerotiorum and $R$. cerealis.

\begin{tabular}{|c|c|c|c|c|}
\hline \multirow{3}{*}{ Compd. } & \multicolumn{4}{|c|}{ Inhibition Rate (\%) } \\
\hline & \multirow{2}{*}{$\begin{array}{c}\text { S. sclerotiorum } \\
50 \mu \mathrm{g} \cdot \mathrm{mL}^{-1}\end{array}$} & \multicolumn{3}{|c|}{ R. cerealis } \\
\hline & & $50 \mu \mathrm{g} \cdot \mathrm{mL}^{-1}$ & $25 \mu \mathrm{g} \cdot \mathrm{mL}^{-1}$ & $12.5 \mu \mathrm{g} \cdot \mathrm{mL}^{-1}$ \\
\hline 12 & $-a$ & - & 95.8 & 60.6 \\
\hline 14 & - & - & 77.5 & 47.9 \\
\hline 17 & 21.4 & 23.6 & - & - \\
\hline
\end{tabular}

\section{Materials and Methods}

\subsection{Instruments}

NMR spectra were obtained at $500 \mathrm{MHz}$ using a Bruker avance III 500 spectrometer (Bruker Daltonics, Bremen, Germany) in $\mathrm{CDCl}_{3}$ or DMSO- $\mathrm{d}_{6}$ solution with tetramethylsilane as the internal standard. HRMS data were obtained on an Ultraflex III MALDI-TOF/TOF (Bruker Daltonics). The melting points were determined on an X-4 binocular microscope melting point apparatus (Beijing Tech Instruments Co., Beijing, China) and are uncorrected.

\subsection{General Synthesis}

All anhydrous solvents were dried and purified by standard techniques. Chemical materials purchased from Aladdin (Shanghai, China) or TCI (Shanghai, China) for analytical grade.

\subsection{General Procedure for the Synthesis of Compounds 1-16}

Unsaturated aldehydes or ketones and $N$-amino-maleimide products were prepared according to the literature $[9,10]$. A mixture of unsaturated aldehydes or ketones $(3.0 \mathrm{mmol}), \mathrm{N}$-amino-maleimide products $(2 \mathrm{mmol})$ and $p$-toluenesulfonic acid $(0.2 \mathrm{mmol})$ in dry ethanol $(5 \mathrm{~mL})$ was heated under reflux for $12 \mathrm{~h}$ with stirring. The organic layer diluted with EtOAc $(20 \mathrm{~mL})$, washed twice with a saturated $\mathrm{NaHCO}_{3}$ solution $(20 \mathrm{~mL})$ and brine $(20 \mathrm{~mL})$, dried with anhydrous $\mathrm{Na}_{2} \mathrm{SO}_{4}$ and evaporated in vacuo. Finally, the residue was purified by silica gel column chromatography (EtOAc/petroleum ether) to afford compounds 1-16.

1-(((E)-4-phenylbut-3-en-2-ylidene)amino)-1H-pyrrole-2,5-dione (1). Pale yellow solid, mp 159-161 ${ }^{\circ} \mathrm{C}$, yield $=67 \% .{ }^{1} \mathrm{H}-\mathrm{NMR}\left(\mathrm{CDCl}_{3}\right) \delta 7.37-7.29(\mathrm{~m}, 5 \mathrm{H}), 6.89(\mathrm{~d}, J=10.2 \mathrm{~Hz}, 1 \mathrm{H}), 6.80(\mathrm{~d}, J=10.2 \mathrm{~Hz}$, 1H), $6.06(\mathrm{~s}, 1 \mathrm{H}), 5.39(\mathrm{~s}, 1 \mathrm{H}), 2.53(\mathrm{~s}, 3 \mathrm{H}) ;{ }^{13} \mathrm{C}-\mathrm{NMR}\left(\mathrm{CDCl}_{3}\right) \delta 154.1,154.0,137.0,136.3,135.8,134.2$, 128.9, 127.2, 110.8, 77.4, 77.1, 76.8, 15.2; HRMS (ESI): $m / z$ calcd for $\mathrm{C}_{14} \mathrm{H}_{12} \mathrm{~N}_{2} \mathrm{O}_{2}[\mathrm{M}+\mathrm{H}]^{+}:$241.0977, found 241.0972 .

1-(((E)-4-(3,5-bis(trifluoromethyl)phenyl)but-3-en-2-ylidene)amino)-1H-pyrrole-2,5-dione (2). Pale yellow solid, $\mathrm{mp} 122-125{ }^{\circ} \mathrm{C}$, yield $=57 \% .{ }^{1} \mathrm{H}-\mathrm{NMR}\left(\mathrm{CDCl}_{3}\right) \delta 7.84(\mathrm{~s}, 1 \mathrm{H}), 7.78(\mathrm{~s}, 2 \mathrm{H}), 6.94(\mathrm{~d}, J=10.2 \mathrm{~Hz}$, $1 \mathrm{H}), 6.83(\mathrm{~d}, J=10.2 \mathrm{~Hz}, 1 \mathrm{H}), 6.17(\mathrm{~s}, 1 \mathrm{H}), 5.36(\mathrm{~s}, 1 \mathrm{H}), 2.57(\mathrm{~s}, 3 \mathrm{H}) ;{ }^{13} \mathrm{C}-\mathrm{NMR}\left(\mathrm{CDCl}_{3}\right) \delta$ 154.3, 154.0, 
138.8, 138.6, 136.9, 133.9, 127.8, 123.0, 108.4, 77.3, 77.1, 76.8, 65.7, 15.2; HRMS (ESI): $m / z$ calcd for $\mathrm{C}_{16} \mathrm{H}_{10} \mathrm{~N}_{2} \mathrm{O}_{2} \mathrm{~F}_{6}[\mathrm{M}+\mathrm{H}]^{+}: 377.0719$, found 377.0699.

1-(((E)-4-(2,4-dichlorophenyl)but-3-en-2-ylidene)amino)-1H-pyrrole-2,5-dione (3). Pale yellow solid, mp 143-144 ${ }^{\circ} \mathrm{C}$, yield $=61 \% .{ }^{1} \mathrm{H}-\mathrm{NMR}\left(\mathrm{CDCl}_{3}\right) \delta 7.40(\mathrm{~s}, 1 \mathrm{H}), 7.22(\mathrm{~d}, J=8.4 \mathrm{~Hz}, 1 \mathrm{H}), 7.01(\mathrm{~d}, J=8.4 \mathrm{~Hz}$, $1 \mathrm{H}), 6.95(\mathrm{~d}, J=10.2 \mathrm{~Hz}, 1 \mathrm{H}), 6.86(\mathrm{~d}, J=10.2 \mathrm{~Hz}, 1 \mathrm{H}), 6.38(\mathrm{~s}, 1 \mathrm{H}), 5.39(\mathrm{~s}, 1 \mathrm{H}), 2.48(\mathrm{~s}, 3 \mathrm{H})$; ${ }^{13} \mathrm{C}-\mathrm{NMR}\left(\mathrm{CDCl}_{3}\right) \delta 153.9,137.7,136.7,134.8,133.7,132.9,131.8,130.1,127.9,108.6,77.3,77.1,76.8,15.1$; HRMS (ESI): $m / z$ calcd for $\mathrm{C}_{14} \mathrm{H}_{10} \mathrm{~N}_{2} \mathrm{O}_{2} \mathrm{Cl}_{2}[\mathrm{M}+\mathrm{H}]^{+}:$308.0113, found 308.0096.

1-(((E)-4-(4-nitrophenyl)but-3-en-2-ylidene)amino)-1H-pyrrole-2,5-dione (4). Pale yellow solid, $\mathrm{mp} 172-174{ }^{\circ} \mathrm{C}$, yield $=52 \% .{ }^{1} \mathrm{H}-\mathrm{NMR}\left(\mathrm{CDCl}_{3}\right) \delta 8.19(\mathrm{~d}, J=8.2 \mathrm{~Hz}, 2 \mathrm{H}), 7.48(\mathrm{~d}, J=8.2 \mathrm{~Hz}, 2 \mathrm{H}), 6.92(\mathrm{~d}, J=10.2 \mathrm{~Hz}$, $1 \mathrm{H}), 6.82(\mathrm{~d}, J=10.2 \mathrm{~Hz}, 1 \mathrm{H}), 6.13(\mathrm{~s}, 1 \mathrm{H}), 5.36(\mathrm{~s}, 1 \mathrm{H}), 2.53(\mathrm{~s}, 3 \mathrm{H}) ;{ }^{13} \mathrm{C}-\mathrm{NMR}\left(\mathrm{CDCl}_{3}\right) \delta$ 154.1, 153.9, 148.1, 142.9, 138.3, 136.8, 133.9, 128.2, 124.2, 109.0, 77.4, 77.2, 76.9, 15.2; HRMS (ESI): $m / z$ calcd for $\mathrm{C}_{14} \mathrm{H}_{11} \mathrm{~N}_{3} \mathrm{O}_{4}[\mathrm{M}+\mathrm{H}]^{+}:$285.0750, found 285.0760.

1-(((E)-4-(2-fluorophenyl)but-3-en-2-ylidene)amino)-1H-pyrrole-2,5-dione (5). Pale yellow solid, $\mathrm{mp} 135-137^{\circ} \mathrm{C}$, yield $=63 \% . \quad{ }^{1} \mathrm{H}-\mathrm{NMR}\left(\mathrm{CDCl}_{3}\right) \delta 7.31-7.26(\mathrm{~m}, 1 \mathrm{H}), 7.10(\mathrm{ddd}, J=29.9,16.0,7.0 \mathrm{~Hz}, 3 \mathrm{H})$, $6.92(\mathrm{~d}, J=10.2 \mathrm{~Hz}, 1 \mathrm{H}), 6.84(\mathrm{~d}, J=10.2 \mathrm{~Hz}, 1 \mathrm{H}), 6.29(\mathrm{~s}, 1 \mathrm{H}), 5.38(\mathrm{~s}, 1 \mathrm{H}), 2.49(\mathrm{~s}, 3 \mathrm{H}) ;{ }^{13} \mathrm{C}-\mathrm{NMR}$ $\left(\mathrm{CDCl}_{3}\right) \delta 161.23,159.3,154.0,137.4,136.5,133.8,130.3,127.7,124.6,116.2,116.0,109.3,77.4,77.1,76.8$, 15.1; HRMS (ESI): $m / z$ calcd for $\mathrm{C}_{14} \mathrm{H}_{11} \mathrm{~N}_{2} \mathrm{O}_{4} \mathrm{~F}[\mathrm{M}+\mathrm{H}]^{+}:$258.0799, found 258.0771.

3-methyl-1-(((E)-4-phenylbut-3-en-2-ylidene)amino)-1H-pyrrole-2,5-dione (6). Pale yellow solid, mp 143-144 ${ }^{\circ} \mathrm{C}$, yield $=68 \% . \quad{ }^{1} \mathrm{H}-\mathrm{NMR}\left(\mathrm{CDCl}_{3}\right) \delta 7.38-7.28(\mathrm{~m}, 5 \mathrm{H}), 6.71(\mathrm{~d}, J=26.2 \mathrm{~Hz}, 1 \mathrm{H})$, $6.04(\mathrm{~s}, 1 \mathrm{H}), 5.35(\mathrm{~d}, J=21.6 \mathrm{~Hz}, 1 \mathrm{H}), 2.53(\mathrm{~d}, J=8.0 \mathrm{~Hz}, 3 \mathrm{H}), 2.13(\mathrm{~d}, J=30.7 \mathrm{~Hz}, 3 \mathrm{H}) ;{ }^{13} \mathrm{C}-\mathrm{NMR}$ $\left(\mathrm{CDCl}_{3}\right) \delta 155.1,154.4,146.0,144.4,137.0,136.2,132.2,130.4,129.0,128.6,127.4,127.1,111.0,110.6$, 77.4, 77.1, 76.9, 16.9, 16.4, 15.4, 15.2; HRMS (ESI): $m / z$ calcd for $\mathrm{C}_{15} \mathrm{H}_{14} \mathrm{~N}_{2} \mathrm{O}_{2}[\mathrm{M}+\mathrm{H}]^{+}: 277.0974$, found 277.0969 .

1-(((E)-4-(3,5-bis(trifluoromethyl)phenyl)but-3-en-2-ylidene)amino)-3-methyl-1H-pyrrole-2,5-dione (7). Pale yellow solid, mp 143-144 ${ }^{\circ} \mathrm{C}$, yield $=68 \% .{ }^{1} \mathrm{H}-\mathrm{NMR}\left(\mathrm{CDCl}_{3}\right) \delta 7.83(\mathrm{~s}, 1 \mathrm{H}), 7.77(\mathrm{~d}, J=5.8 \mathrm{~Hz}, 2 \mathrm{H})$, $6.74(\mathrm{~d}, J=40.7 \mathrm{~Hz}, 1 \mathrm{H}), 6.15(\mathrm{~s}, 1 \mathrm{H}), 5.33(\mathrm{~d}, J=21.8 \mathrm{~Hz}, 1 \mathrm{H}), 2.56(\mathrm{~d}, J=9.1 \mathrm{~Hz}, 3 \mathrm{H}), 2.16(\mathrm{~d}, J=37.4 \mathrm{~Hz}$, 3H); ${ }^{13} \mathrm{C}-\mathrm{NMR}\left(\mathrm{CDCl}_{3}\right) \delta$ 155.4, 155.0, 154.7, 154.3, 146.9, 144.3, 139.1, 138.7, 132.8, 132.4, 132.1, 130.0, 127.8, 124.2, 122.9, 122.0, 108.7, 107.9, 77.4, 76.9, 65.8, 65.4, 17.0, 16.4, 15.6, 15.4; HRMS (ESI): $\mathrm{m} / \mathrm{z}$ calcd for $\mathrm{C}_{17} \mathrm{H}_{12} \mathrm{~N}_{2} \mathrm{O}_{2} \mathrm{~F}_{6}[\mathrm{M}+\mathrm{H}]^{+}$: 391.0875, found 391.0904.

1-(((E)-4-(2,4-dichlorophenyl)but-3-en-2-ylidene)amino)-3-methyl-1H-pyrrole-2,5-dione (8). Pale yellow solid, mp 143-144 ${ }^{\circ} \mathrm{C}$, yield $=68 \% .{ }^{1} \mathrm{H}-\mathrm{NMR}\left(\mathrm{CDCl}_{3}\right) \delta 7.39(\mathrm{t}, J=2.0 \mathrm{~Hz}, 1 \mathrm{H}), 7.21(\mathrm{ddd}, J=8.3,4.9,2.1 \mathrm{~Hz}$, $1 \mathrm{H}), 7.01(\mathrm{dd}, J=12.4,8.4 \mathrm{~Hz}, 1 \mathrm{H}), 6.76(\mathrm{~d}, J=25.1 \mathrm{~Hz}, 1 \mathrm{H}), 6.37(\mathrm{~d}, J=20.2 \mathrm{~Hz}, 1 \mathrm{H}), 5.35(\mathrm{~d}, J=37.7 \mathrm{~Hz}$, $1 \mathrm{H}), 2.47(\mathrm{~d}, J=5.7 \mathrm{~Hz}, 3 \mathrm{H}), 2.16(\mathrm{~d}, J=32.3 \mathrm{~Hz}, 3 \mathrm{H}) ;{ }^{13} \mathrm{C}-\mathrm{NMR}\left(\mathrm{CDCl}_{3}\right) \delta 155.0,154.3,146.6,144.1$, 137.8, 134.8, 133.2, 132.9, 132.6, 132.2, 130.3, 129.9, 128.2, 127.9, 108.9, 108.1, 77.4, 77.1, 76.9, 17.0, 16.4, 15.3, 15.1; HRMS (ESI): $m / z$ calcd for $\mathrm{C}_{15} \mathrm{H}_{12} \mathrm{~N}_{2} \mathrm{O}_{2} \mathrm{Cl}_{2}[\mathrm{M}+\mathrm{H}]^{+}: 323.0348$, found 323.0355.

4,5,6,7-tetrachloro-2-(((E)-4-phenylbut-3-en-2-ylidene)amino)isoindoline-1,3-dione (9). Pale yellow solid, $\mathrm{mp} 212-213{ }^{\circ} \mathrm{C}$, yield $=58 \% .{ }^{1} \mathrm{H}-\mathrm{NMR}\left(\mathrm{CDCl}_{3}\right) \delta 7.56(\mathrm{~d}, J=7.2 \mathrm{~Hz}, 2 \mathrm{H}), 7.47-7.34(\mathrm{~m}, 3 \mathrm{H})$, $7.31(\mathrm{~d}, J=16.5 \mathrm{~Hz}, 1 \mathrm{H}), 7.17(\mathrm{~d}, J=16.4 \mathrm{~Hz}, 1 \mathrm{H}), 2.20(\mathrm{~s}, 3 \mathrm{H}) ;{ }^{13} \mathrm{C}-\mathrm{NMR}\left(\mathrm{CDCl}_{3}\right) \delta$ 176.1, 159.6, 140.5, 135.2, 130.0, 129.1, 127.6, 127.1, 126.9, 77.4, 77.1, 76.8, 16.4; HRMS (ESI): $m / z$ calcd for $\mathrm{C}_{18} \mathrm{H}_{10} \mathrm{~N}_{2} \mathrm{O}_{2} \mathrm{Cl}_{4}$ $[\mathrm{M}+\mathrm{H}]^{+}:$448.9388, found 448.9383 .

3,4-dichloro-1-(((E)-4-phenylbut-3-en-2-ylidene)amino)-1H-pyrrole-2,5-dione (10). Pale yellow solid, $\mathrm{mp} 152-153^{\circ} \mathrm{C}$, yield $=62 \% .{ }^{1} \mathrm{H}-\mathrm{NMR}\left(\mathrm{CDCl}_{3}\right)$ 8 7.39-7.31 (m, 5H), $6.10(\mathrm{~s}, 1 \mathrm{H}), 5.49(\mathrm{~s}, 1 \mathrm{H}), 2.56(\mathrm{~s}, 3 \mathrm{H})$; 
${ }^{13} \mathrm{C}-\mathrm{NMR}\left(\mathrm{CDCl}_{3}\right) \delta$ 149.5, 149.2, 139.4, 137.9, 137.3, 134.6, 129.3, 129.0, 127.7, 111.6, 77.4, 77.1, 76.9, 15.3; HRMS (ESI): $m / z$ calcd for $\mathrm{C}_{14} \mathrm{H}_{10} \mathrm{~N}_{2} \mathrm{O}_{2} \mathrm{Cl}_{2}[\mathrm{M}+\mathrm{H}]^{+}:$308.0113, found 308.0093 .

1-(((E)-4-(3,5-bis(trifluoromethyl)phenyl)but-3-en-2-ylidene)amino)-3,4-dichloro-1H-pyrrole-2,5-dione (11). Pale yellow solid, $\mathrm{mp} 152-153{ }^{\circ} \mathrm{C}$, yield $=60 \% .{ }^{1} \mathrm{H}-\mathrm{NMR}\left(\mathrm{CDCl}_{3}\right) \delta 7.87(\mathrm{~s}, 1 \mathrm{H}), 7.81(\mathrm{~s}, 2 \mathrm{H}), 6.22(\mathrm{~s}, 1 \mathrm{H})$, $5.47(\mathrm{~s}, 1 \mathrm{H}), 2.60(\mathrm{~s}, 3 \mathrm{H}) ;{ }^{13} \mathrm{C}-\mathrm{NMR}\left(\mathrm{CDCl}_{3}\right) \delta 150.0,149.2,140.2,139.2,137.8,137.5,132.7,132.4,128.4$, 123.5, 109.3, 77.4, 77.2, 76.9, 15.5; HRMS (ESI): $m / z$ calcd for $\mathrm{C}_{16} \mathrm{H}_{8} \mathrm{~N}_{2} \mathrm{O}_{2} \mathrm{~F}_{6} \mathrm{Cl}_{2}[\mathrm{M}+\mathrm{H}]^{+}: 466.9759$, found 466.9751 .

3,4-dichloro-1-(((E)-4-(2,4-dichlorophenyl)but-3-en-2-ylidene)amino)-1H-pyrrole-2,5-dione (12). Pale yellow solid, $\mathrm{mp} 189-191{ }^{\circ} \mathrm{C}$, yield $=62 \% .{ }^{1} \mathrm{H}-\mathrm{NMR}\left(\mathrm{CDCl}_{3}\right) \delta 7.42(\mathrm{~d}, J=2.0 \mathrm{~Hz}, 1 \mathrm{H}), 7.27-7.25(\mathrm{~m}, 1 \mathrm{H})$, $7.05(\mathrm{~d}, J=8.4 \mathrm{~Hz}, 1 \mathrm{H}), 6.45(\mathrm{~s}, 1 \mathrm{H}), 5.47(\mathrm{~s}, 1 \mathrm{H}), 2.52(\mathrm{~s}, 3 \mathrm{H}) ;{ }^{13} \mathrm{C}-\mathrm{NMR}\left(\mathrm{CDCl}_{3}\right) \delta$ 155.0, 154.3, 146.6, 144.1, 137.8, 134.8, 133.2, 132.9, 132.6, 132.2, 130.3, 129.9, 128.2, 127.9, 108.9, 108.1, 77.4, 77.1, 76.8, 16.4, 15.3, 15.1; HRMS (ESI): $m / z$ calcd for $\mathrm{C}_{14} \mathrm{H}_{8} \mathrm{~N}_{2} \mathrm{O}_{2} \mathrm{Cl}_{4}[\mathrm{M}+\mathrm{H}]^{+}: 398.9232$, found 398.9234 .

3,4-dichloro-1-(((E)-4-(4-nitrophenyl)but-3-en-2-ylidene)amino)-1H-pyrrole-2,5-dione (13). Pale yellow solid, mp 161-163 ${ }^{\circ} \mathrm{C}$, yield $=41 \% .{ }^{1} \mathrm{H}-\mathrm{NMR}\left(\mathrm{CDCl}_{3}\right) \delta 8.22(\mathrm{~s}, 2 \mathrm{H}), 7.52(\mathrm{~d}, J=8.5 \mathrm{~Hz}, 2 \mathrm{H}), 6.20(\mathrm{~s}, 1 \mathrm{H})$, $5.48(\mathrm{~s}, 1 \mathrm{H}), 2.57(\mathrm{~s}, 3 \mathrm{H}) ;{ }^{13} \mathrm{C}-\mathrm{NMR}\left(\mathrm{CDCl}_{3}\right) \delta 154.1,153.9,148.1,142.9,138.3,136.8,133.9,128.2$, 124.2, 109.0, 77.4, 77.2, 76.9, 15.2; HRMS (ESI): $m / z$ calcd for $\mathrm{C}_{14} \mathrm{H}_{9} \mathrm{~N}_{3} \mathrm{O}_{4} \mathrm{Cl}_{2}[\mathrm{M}+\mathrm{H}]^{+}: 352.9956$, found 352.9970 .

3,4-dichloro-1-(((E)-4-(2-fluorophenyl)but-3-en-2-ylidene)amino)-1H-pyrrole-2,5-dione (14). Pale yellow solid, $\mathrm{mp} 140-141{ }^{\circ} \mathrm{C}$, yield $=64 \% .{ }^{1} \mathrm{H}-\mathrm{NMR}\left(\mathrm{CDCl}_{3}\right) \delta 7.33-7.29(\mathrm{~m}, 1 \mathrm{H}), 7.18-7.13(\mathrm{~m}, 2 \mathrm{H}), 7.07(\mathrm{~s}, 1 \mathrm{H})$, $6.33(\mathrm{~s}, 1 \mathrm{H}), 5.47(\mathrm{~s}, 1 \mathrm{H}), 2.53(\mathrm{~d}, J=1.5 \mathrm{~Hz}, 3 \mathrm{H}) ;{ }^{13} \mathrm{C}-\mathrm{NMR}\left(\mathrm{CDCl}_{3}\right) \delta 161.5,159.5,149.3,139.7$, 137.7, 130.8, 128.2, 124.8, 122.0, 116.4, 116.2, 110.2, 77.4, 77.1, 76.9, 15.3; HRMS (ESI): $m / z$ calcd for $\mathrm{C}_{14} \mathrm{H}_{9} \mathrm{~N}_{2} \mathrm{O}_{2} \mathrm{Cl}_{2} \mathrm{~F}[\mathrm{M}+\mathrm{H}]^{+}: 326.0019$, found 325.9990 .

3,4-dichloro-1-(((E)-4-(p-tolyl)but-3-en-2-ylidene)amino)-1H-pyrrole-2,5-dione (15). Pale yellow solid, mp 123-124 ${ }^{\circ} \mathrm{C}$, yield $=65 \% .{ }^{1} \mathrm{H}-\mathrm{NMR}\left(\mathrm{CDCl}_{3}\right) \delta 7.22(\mathrm{~d}, J=7.8 \mathrm{~Hz}, 2 \mathrm{H}), 7.17(\mathrm{~d}, J=7.7 \mathrm{~Hz}, 2 \mathrm{H})$, $6.08(\mathrm{~s}, 1 \mathrm{H}), 5.47(\mathrm{~s}, 1 \mathrm{H}), 2.56(\mathrm{~s}, 3 \mathrm{H}), 2.33(\mathrm{~s}, 3 \mathrm{H}) ;{ }^{13} \mathrm{C}-\mathrm{NMR}\left(\mathrm{CDCl}_{3}\right) \delta 149.6,149.3,139.4,138.1,137.3$, 131.6, 129.7, 127.8, 111.8, 77.4, 77.2, 76.9, 29.8, 15.4; HRMS (ESI): $m / z$ calcd for $\mathrm{C}_{15} \mathrm{H}_{12} \mathrm{~N}_{2} \mathrm{O}_{2} \mathrm{Cl}_{2}[\mathrm{M}+\mathrm{H}]^{+}$: 345.0168 , found 345.0170 .

3,4-dichloro-1-(((E)-4-(2,6-dichlorophenyl)but-3-en-2-ylidene)amino)-1H-pyrrole-2,5-dione (16). Pale yellow solid, mp 212-213 ${ }^{\circ} \mathrm{C}$, yield $=59 \% .{ }^{1} \mathrm{H}-\mathrm{NMR}\left(\mathrm{CDCl}_{3}\right) \delta 7.40(\mathrm{~d}, J=7.7 \mathrm{~Hz}, 1 \mathrm{H}), 7.28-7.19(\mathrm{~m}, 2 \mathrm{H}), 6.97(\mathrm{~s}, 1 \mathrm{H})$, $5.34(\mathrm{~s}, 1 \mathrm{H}), 2.53(\mathrm{~s}, 3 \mathrm{H}) ;{ }^{13} \mathrm{C}-\mathrm{NMR}\left(\mathrm{CDCl}_{3}\right) \delta 149.5,149.2,139.8,139.6,137.2,136.0,133.6,130.4,129.2$, 128.2, 107.0, 77.4, 77.2, 76.9, 15.3; HRMS (ESI): $m / z$ calcd for $\mathrm{C}_{14} \mathrm{H}_{8} \mathrm{~N}_{2} \mathrm{O}_{2} \mathrm{Cl}_{4}[\mathrm{M}+\mathrm{H}]^{+}: 398.9232$, found 398.9260 .

\subsection{Synthesis of (E)-2-(1-hydroxy-3-phenylallylidene)-4,5-dimethoxycyclopent-4-ene-1,3-dione (Linderone)}

This compound was prepared according to literature [11]: yield $=84 \%$. NMR data consistent with literature [11]: ${ }^{1} \mathrm{H}-\mathrm{NMR}\left(\mathrm{CDCl}_{3}\right) \delta 11.56(\mathrm{~s}, 1 \mathrm{H}), 7.67(\mathrm{~s}, 2 \mathrm{H}), 7.62(\mathrm{~m}, 2 \mathrm{H}), 7.41(\mathrm{~m}, 3 \mathrm{H}), 4.22(\mathrm{~s}, 3 \mathrm{H})$, $4.17(\mathrm{~s}, 3 \mathrm{H})$.

\subsection{Synthesis of (E)-4,5-dimethoxy-2-(1-methoxy-3-phenylallylidene)cyclopent-4-ene-1,3-dione} (Methyllinderone)

Methyllinderone was prepared from linderone according to the literature [11]. Yield $=93 \%$; ${ }^{1} \mathrm{H}-\mathrm{NMR}\left(\mathrm{CDCl}_{3}\right) \delta 7.94(\mathrm{~d}, J=15.6 \mathrm{~Hz}, 1 \mathrm{H}), 7.55-7.65(\mathrm{~m}, 2 \mathrm{H}), 7.52(\mathrm{~d}, J=15.6 \mathrm{~Hz}, 1 \mathrm{H}), 7.30-7.40$ $(\mathrm{m}, 3 \mathrm{H}), 4.19(\mathrm{~s}, 6 \mathrm{H}), 4.10(\mathrm{~s}, 3 \mathrm{H})$. 


\subsection{General Procedure for the Synthesis of Copound $\mathbf{1 7}$}

3,4-dichloro-1H-pyrrole-2,5-dione (17). The compound $\mathbf{1 7}$ was prepared according to the literature [12]. Orange crystal powder, mp $178-179{ }^{\circ} \mathrm{C}$, yield $=82 \% .{ }^{1} \mathrm{H}-\mathrm{NMR}\left(500 \mathrm{MHz}, \mathrm{DMSO}-d_{6}\right)$ $\delta 11.73(\mathrm{~s}, 1 \mathrm{H}) ;{ }^{13} \mathrm{C}-\mathrm{NMR}\left(126 \mathrm{MHz}, \mathrm{DMSO}-d_{6}\right) \delta 164.48,133.26$. HRMS (ESI): $m / z$ calcd for $\mathrm{C}_{4} \mathrm{HNO}_{2} \mathrm{Cl}_{2}$ $[\mathrm{M}+\mathrm{H}]^{+}:$163.9312, found 163.9306 .

\subsection{Insecticidal Biological Assay}

All bioassays were performed on representative test organisms reared in the laboratory. The bioassay was repeated at $25 \pm 1^{\circ} \mathrm{C}$ according to statistical requirements. Assessments were made on a dead/alive basis and mortality rates were corrected using Abbott's formula [13]. Evaluations are based on a percentage scale of $0-100$ in which $0=$ no activity and $100=$ total kill.

\subsubsection{Stomach Toxicity against Oriental Armyworm (Mythimna separata)}

The stomach toxicities of compounds 1-8, 10 against oriental armyworm were evaluated by foliar application using the reported procedure [14]. For the foliar armyworm tests, individual corn leaves were placed on moistened pieces of filter paper in Petri dishes. The leaves were then sprayed with the test solution and allowed to dry. The dishes were infested with 10 fourth-instar oriental armyworm larvae. Percentage mortalities were evaluated 3 days after treatment. Each treatment was performed three times.

\subsubsection{Toxicity against Mosquito (Culex pipiens pallens)}

The toxicities of compounds 1-8, 10 against mosquito were evaluated according to the reported procedure $[15,16]$. One milliliter of different concentrated dilutions of each compound was added to $99 \mathrm{~mL}$ of water to obtain different concentrations of tested solution. Then 20 fourth-instar mosquito larvae were put into the solution. Percentage mortalities were evaluated 8 days after treatment. Each test was performed in triplicate.

\subsection{In Vitro Antifungal Bioassay}

The antifungal activities were screened and evaluated by the poison plate technique [17]. All final compounds were dissolved in DMF $(0.1 \mathrm{~mL})$ before mixing with potato dextrose agar (PDA; $9.9 \mathrm{~mL}$ ). The compounds were tested at a concentration of $50 \mu \mathrm{g} \cdot \mathrm{mL}^{-1}$. All fungi were cultivated in PDA at $27 \pm 1^{\circ} \mathrm{C}$ for 4 days to make new mycelium for the identification of antifungal activity. Then, mycelia dishes of approximately $5 \mathrm{~mm}$ diameter were cut from the culture medium. A mycelium was obtained using a germ-free inoculation needle and inoculated in the middle of the PDA plate aseptically. The inoculated plates were incubated at $27 \pm 1^{\circ} \mathrm{C}$ for 5 days. DMF in sterile distilled water served as the negative control, whereas hymexazol served as the positive control. Each treatment condition consisted of three replicates. Radial growth of the fungal colonies was measured, and the data were statistically analyzed. Inhibitory effects of the test compounds in vitro on these fungi were calculated by the formula $I(\%)=[(C-T) /(C-0.5)] \times 100$, where $C$ represents the diameter of fungal growth on untreated PDA, $T$ represents the diameter of fungi on treated PDA, and $I$ represents the inhibition rate.

\section{Conclusions}

A series of novel $\mathrm{N}$-amino-maleimide derivatives containing hydrazone group were designed and synthesized and their insecticidal and antifungal activities were evaluated and discussed. The results of bioassays indicated that some compounds (compounds 2, 3 and 8) possessed excellent activities against mosquito and compounds 2, 3 and $\mathbf{6}$ exhibited considerable activities against oriental armyworm. In particular, some compounds exhibited better antifungal activities against fourteen phytogenic fungi than linderone and methyllinderone. This shows that using natural active substance as a first lead 
structure, designing and synthesizing its derivatives is a good choice for discovering active pesticide molecules. Because compound 12 exhibited best broad-spectrum antifungal activities, it makes sense to further explore its fungicidal mechanism, chitinase inhibitory activity in vitro and discover the fungicide candidate.

Supplementary Materials: The following are available online, Figures S1-S34, NMR spectral information of compounds 1-17.

Author Contributions: R.S. conceived and designed the experiments; X.S., C.L. and P.C. performed the experiments, analyzed the data; X.S. drafted the manuscript; R.S. revised the manuscript; H.Z. gave some suggestion to the experiment. All authors have read and approved the final manuscript.

Funding: This work was financially supported by the National Science Foundation of China (No. 21462028), Startup Foundation for Outstanding Young Scientists of Hainan University (kyqd1640) and Hainan Provincial Natural Science Foundation Innovation Research Team Project (2016CXTD002).

Conflicts of Interest: The authors declare no conflict of interest

\section{References}

1. Chen, Q.; Zhang, J.M.; Chen, L.L.; Yang, J.; Yang, X.L.; Ling, Y.; Yang, Q. Design and synthesis of chitin synthase inhibitors as potent fungicides. Chin. Chem. Lett. 2017, 28, 1232-1237. [CrossRef]

2. Wei, G.; Jiang, H.; Geng, Y. Advances in studies on pharmacological activities of genus lindera. Zhonghua Zhongyiyao Xuekan 2014, 32, 61-62. [CrossRef]

3. Hwang, E.I.; Lee, Y.M.; Lee, S.M.; Yeo, W.H.; Moon, J.S.; Kang, T.H.; Park, K.D.; Kim, S.U. Inhibition of chitin synthase 2 and antifungal activity of lignans from the stem bark of Lindera erythrocarp. Planta Med. 2007, 73, 679-682. [CrossRef] [PubMed]

4. Lee, S.H.; Oh, H.W.; Fang, Y.; An, S.B.; Park, D.S.; Song, H.H.; Oh, S.R.; Kim, S.Y.; Kim, S.; Kim, N.; et al. Identification of plant compounds that disrupt the insect juvenile hormone receptor complex. Proc. Natl. Acad. Sci. USA 2015, 112, 1733-1738. [CrossRef] [PubMed]

5. Wang, S.Y.; Lan, X.Y.; Xiao, J.H.; Yang, J.C.; Kao, Y.T.; Chang, S.T. Antiinflammatory activity of Lindera erythrocarpa fruits. Phytother. Res. 2008, 22, 213-216. [CrossRef] [PubMed]

6. Salewska, N.; Boros-Majewska, J.; Lacka, I.; Chylińska, K.; Sabisz, M.; Milewski, S.; Milewska, M.J. Chemical reactivity and antimicrobial activity of N-substituted maleimides. J. Enzyme Inhib. Med. Chem. 2012, 27, 117-124. [CrossRef] [PubMed]

7. Gholap, A.R.; Toti, K.S.; Shirazi, F.; Kumari, R.; Bhat, M.K.; Deshpande, M.V.; Srinivasan, K.V. Synthesis and evaluation of antifungal properties of a series of the novel 2-amino-5-oxo-4-phenyl-5,6,7,8-tetrahydroquinoline-3-carbonitrile and its analogues. Bioorg. Med. Chem. 2007, 15, 6705-6715. [CrossRef] [PubMed]

8. Long, D.Q.; Li, D.J. Synthesis and bactericidal activity of novel triazolopyrimidine formyl hydrazones. Chem. Reag. 2008, 30, 206-208. [CrossRef]

9. Zumbansen, K.; Döhring, A.; List, B. Morpholinium trifluoroacetate-catalyzed aldol condensation of acetone with both aromatic and aliphatic aldehydes. Adv. Synth. Catal. 2010, 352, 1135-1138. [CrossRef]

10. Ma, X.L.; Huang, J.X.; Duan, W.G.; Mo, Q.J.; Lin, G.S.; Huang, Y. Synthesis and fungicidal activity of a-terpinene-maleimide-based acylhydrazone derivatives. Chin. J. Org. Chem. 2012, 32, 1077-1083. [CrossRef]

11. Xiao, F.; Liu, W.; Wang, Y.; Zhang, Q.; Li, X.; Hu, X. Concise synthesis of linderaspirone, A. and Bi-linderone. Asian J. Org. Chem. 2013, 2, 216-219. [CrossRef]

12. Chen, X.; Zheng, Y.; Shen, Y. Natural products with maleic anhydride structure: Nonadrides, tautomycin, chaetomellic anhydride, and other compounds. Chem. Rev. 2007, 107, 1777-1830. [CrossRef] [PubMed]

13. Abbott, W.S. A method of computing the effectiveness of an insecticide. J. Am. Mosq. Control Assoc. 1987, 3, 302-303. [CrossRef] [PubMed]

14. Zhao, Q.Q.; Shang, J.; Liu, Y.X.; Wang, K.Y.; Bi, F.C.; Huang, R.Q.; Wang, Q.M. Synthesis and insecticidal activities of novel $N$-sulfenyl- $N$-tert-butyl- $N, N^{\prime}$-diacylhydrazines. 1 . N-Alkoxysulfenate derivatives. J. Agric. Food Chem. 2007, 55, 9614-9619. [CrossRef] [PubMed]

15. Zhao, Q.Q.; Li, Y.Q.; Xiong, L.X.; Wang, Q.M. Design, Synthesis and insecticidal activity of novel Phenylpyrazoles containing a 2,2,2-Trichloro-1-alkoxyethyl moiety. J. Agric. Food Chem. 2010, 58, 4992-4998. [CrossRef] [PubMed] 
16. Raymond, M.; Marquine, M. Evolution of insecticide resistance in culex pipiens populations: The corsican paradox. J. Evol. Biol. 1994, 7, 315-337. [CrossRef]

17. Wang, X.; Li, P.; Li, Z.; Yin, J.; He, M.; Xue, W.; Chen, Z.; Song, B. Synthesis and bioactivity evaluation of novel arylimines containing a 3-aminoethyl-2-[( $p$-trifluoromethoxy)anilino]-4(3H)-quinazolinone moiety. J. Agric. Food Chem. 2013, 61, 9575-9582. [CrossRef] [PubMed]

Sample Availability: Not available.

(C) 2018 by the authors. Licensee MDPI, Basel, Switzerland. This article is an open access article distributed under the terms and conditions of the Creative Commons Attribution (CC BY) license (http:// creativecommons.org/licenses/by/4.0/). 